\title{
Contrast-enhanced ultrasonography features of papillary thyroid carcinoma for predicting cervical lymph node metastasis
}

\author{
YING LIU $^{1}$, HONG ZHOU ${ }^{1}$, PENG YANG ${ }^{2}$, YANG ZHOU ${ }^{1}$, JIAN WU ${ }^{3}$, \\ CHANGYU CHEN $^{1}$, MING YE ${ }^{1}$ and JING LUO ${ }^{3}$ \\ Departments of ${ }^{1}$ Ultrasound, ${ }^{2}$ Pathology and ${ }^{3}$ Thyroid Surgery, The Third People's Hospital of Chengdu and \\ The Affiliated Clinical Medical College of Southwest Jiao Tong University, Qingyang, Chengdu, Sichuan 610031, P.R. China
}

Received March 16, 2017; Accepted August 21, 2017

DOI: $10.3892 /$ etm.2017.5087

\begin{abstract}
The present study was performed to evaluate the predictive value of contrast-enhanced ultrasonography (CEUS) for the risk of cervical lymph node metastasis (LNM) in papillary thyroid carcinoma (PTC). Between May 2014 and November 2016, 42 patients who received surgery for suspicious PTCs were enrolled in the present study. Each individual underwent CEUS with conventional ultrasound (US), preoperative US-guided fine needle aspiration and personalized surgery. Subsequently, the microvascular density (MVD) of all surgical specimens was measured. According to the results of surgical histopathology, individuals were divided into two groups: $\mathrm{LNM}^{+}$(PTCs with LNM), and LNM' (PTCs without LNM). Clinicopathological characteristics, CEUS enhancement patterns, perfusion parameters and measurements of MVD were compared. The correlation between quantitative variables and LNM was analyzed using Spearman's correlation analysis. Compared with that in the $\mathrm{LNM}^{-}$group, patients in the $\mathrm{LNM}^{+}$group were younger $(\mathrm{P}<0.05)$ and had a larger mean tumor size $(\mathrm{P}<0.05)$. The incidence ratio of patients who were $\leq 45$ years old $(\mathrm{P}<0.05)$, tumors $>10 \mathrm{~mm}$ in size $(\mathrm{P}<0.05)$ and capsular infiltration $(\mathrm{P}<0.05)$ were statistically higher in the $\mathrm{LNM}^{+}$group. Following the use of a novel classification system, the ratio of PTCs with early partial hyper-enhancement was identified to be significantly higher in the $\mathrm{LNM}^{+}$group $(\mathrm{P}<0.01)$. The mean intensity, intensity increase velocity, MVD ratio and mean intensity ratio of intratumoral/peripheral thyroid parenchyma (MIR) were
\end{abstract}

Correspondence to: Professor Hong Zhou, Department of Ultrasound, The Third People's Hospital of Chengdu and The Affiliated Clinical Medical College of Southwest Jiao Tong University, 82 Qinglong Street, Qingyang, Chengdu, Sichuan 610031, P.R. China

E-mail: 664121529@qq.com

Key words: contrast-enhanced ultrasonography, papillary thyroid carcinoma, fine needle aspiration, lymph node metastasis, microvascular density, mean intensity ratio of intratumoral/peripheral thyroid parenchyma statistically higher in the $\mathrm{LNM}^{+}$group compared with that in the $\mathrm{LNM}^{-}$group (all $\mathrm{P}<0.05$ ). MIR was identified to be positive correlated with LNM $(\mathrm{P}<0.05)$. A MIR value of 0.86 was the optimal threshold of LNM in PTCs. In conclusion, LNM may rely on the local rich blood supply of PTC lesions. Partial hyper-enhancements of CEUS and higher values of MIR may suggest a high risk for LNM in PTC.

\section{Introduction}

Papillary thyroid cancer (PTC) is the most common type of well-differentiated thyroid cancer (1). Primary lymph node metastasis (LNM) occurs in approximately 30-40\% of cases, and microscopic involvement of the lymph nodes may occur in as high as $80-90 \%$ (2). LNM is an important risk indicator of locoregional recurrence, and regional LNM could be the second most important independent prognostic indicator (3-5). Identifying the characteristics of PTCs for invasion and metastasis, is helpful for clinicians to assesse the risk of recurrent disease and to provide a personalized therapy for each patient. Patients with PTC have good prognosis after successfully personalized management, with the reported 10-year survival rate recorded at 93\% (6). Recent researches have focused on how to reduce excess therapy and screen high-risk patients $(7,8)$. Fine needle aspiration (FNA) is an important diagnostic tool in the evaluation of thyroid nodules. The 2015 ATA guidelines strongly recommend FNA for nodules that appear suspicious during ultrasound (US) (9). However, FNA cannot provide accurate evaluation of tumor features for invasion and metastasis. Even though the expression of genes in PTCs involved in tumor angiogenesis and metastasis has been investigated recently $(10,11)$, the main constraint on its clinical application is the high cost. It is necessary to establish a more promising diagnostic modality concerning optimization of cost-effectiveness and perioperative prediction of lymph nodes clearance.

LNM in PTCs usually occurs initially in the central cervical lymph node compartment ahead of the lateral compartment, in spite of their occasional skip to the lateral compartment, leaving the central compartment tumor-free $(12,13)$. US has been widely used for preoperative staging in patients with PTC to determine the necessary extent of surgery. Yet, LNM in the central neck may not appear abnormal on preoperative US detection, the 
ability of preoperative US to identify central neck LNM is limited (14). Therefore, a growing number of researchers are advocating prophylactic central lymph node dissection (CLND) for PTC patients staging cNO (AJCC/UICC TNM, 7th edition, as proposed by the College of American Pathologists) based on a preoperative US or upon cervical palpation $(15,16)$. However, controversy whether prophylactic CLND has major impact on disease-specific survival still exists (17). Prophylactic CLND might benefit greatly if PTC patients with high risk of LNM can be screened preoperatively. Tumor angiogenesis or neovascularization is accepted as an indicator of tumor growth and metastasis (18-20). A recent study demonstrated higher blood supply is associated with regional LNM of PTC (21), while studies of microvascular density (MVD) have generated a conflicting hypothesis that recurrent thyroid cancers expressed less intratumoral microvessels (22). Given that by providing visualization of the macro- and microvascularization of the tumor, contrast-enhanced US (CEUS) can evaluate thyroid tumors qualitatively and quantitatively. Though some studies have evaluated the value of CEUS in the diagnosis of thyroid malignant nodules $(23,24)$, there are few studies available assessing CEUS to help risk-stratify PTC patients with LNM. In this study we investigated the clinical implications of CEUS in PTCs, and examined the relation between enhancements of PTC and the risk of LNM.

\section{Patients and methods}

Patients. The present study's design and protocol were approved by the Ethics Committee of the Third People's Hospital of Chengdu (Sichuan, China) and all study subjects signed an informed consent. A retrospective analysis of prospectively collected data was performed, from May 2014 to November 2016, based on 42 patients candidate to surgery for suspicious PTCs. Subjects with the following US-based detection criteria were included: suspicious PTCs including sonographic findings: solid hypoechoic, long/short ratio $<2$, poorly defined margin, and microcalcifications. The exclusion criteria were: i) a nodule with a fluid content $>50 \%$ of volume; ii) the presence of a coarse calcification inside a nodule; iii) insufficient normal thyroid tissue around the target nodule; iv) any condition of hyperthyroidism; v) pregnancy; vi) heart failure and vii) severe pulmonary hypertension (25). The study population that was enrolled for investigation comprised a total of 42 individuals, including 33 women and 9 men (mean age, 49.1 years; range, $22-73$ years; $S D \pm 13.5$ years). The individuals were advised that any surgical procedure would not be influenced by participation in the present study.

CEUS. Once enrolled in the study, the individuals underwent CEUS with conventional US by 2 experienced radiologists 3 days before any surgical intervention, using an IU Elite scanner (Phillips Medical Systems, Bothell, WA, USA), equipped with an L12-5 transducer. Initially, the 3 dimensions of the nodules were measured by US, and the largest diameter was recorded as the nodule size. CEUS was performed to examine the mass or suspicious region. Under the US contrast mode the mechanical index (MI) ranged between 0.06 and 0.08 , and the focal point was adjusted to the middle or the lower edge of the thyroid nodules. Double-contrast mode

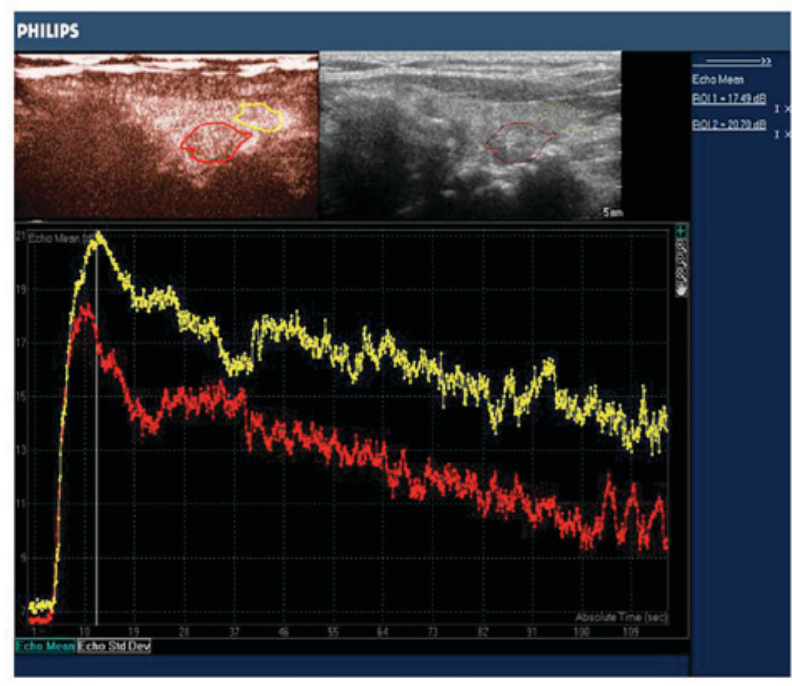

Figure 1. ROI and time-intensity curves in a PTC lesion (red tracing) and the PTC (yellow tracing), respectively. ROI, region of interest; PTC, papillary thyroid carcinoma.

was used to ensure the probe was in parallel with the long axis of the thyroid gland as much as possible. If there were multifocal lesions, the most suspicious lesion was taken for analysis. The contrast medium was obtained by adding $5 \mathrm{ml}$ of physiological saline to SonoVue (Bracco SpA, Milan, Italy). Once the largest plane of the lesion in long axis was selected, a contrast bolus of $2.4 \mathrm{ml}$ was injected manually via a 20 -gauge intravenous cannula placed in an antecubital vein, and $5 \mathrm{ml}$ of normal saline followed as a flush. Meanwhile, the timer on the US system was started, and the imaging plane was kept as stable as possible. Each contrast imaging acquisition lasted $\geq 3$ min after bolus injection and was digitally stored. The imaging data obtained from CEUS were continuously stored. All individuals were monitored for adverse events until $20 \mathrm{~min}$ after the procedure. The offline analysis was performed with dedicated software (Q-LAB ${ }^{\circledR}$; Philips Medical Systems, The Netherlands). In each case, based upon CEUS, signal intensity changes of the nodule were measured in decibels (dB). A region of interest (ROI) was traced out along the edge of the nodule, and another ROI of similar size on the peripheral thyroid parenchyma (Fig. 1). Quantitative perfusion parameters were obtained using the ROI method and time-intensity curves, including baseline signal intensity (BI, dB), Time of enhancement beginning (TEB, sec), time to peak (TP, sec), peak accelerating time (PAT, sec), peak intensity (PI, dB), mean intensity (MI, dB), intensity increase velocity (IIV, $\mathrm{dB} / \mathrm{sec}$ ), and intensity decrease velocity (IDV, $\mathrm{dB} / \mathrm{sec})$.

US-guided FNA. Based on CEUS and US imaging, preoperative US-guided FNA was performed to confirm the cytological diagnosis in 42 cases. The FNA procedure was carried out using a standard 21 gauge needle. Sampling typically targets the solid component of the lesion. If there was more than one nodule, a sample was taken from the nodule with suspicious or atypical US characteristics. The cytopathological system used in the present study was the Bethesda system. 

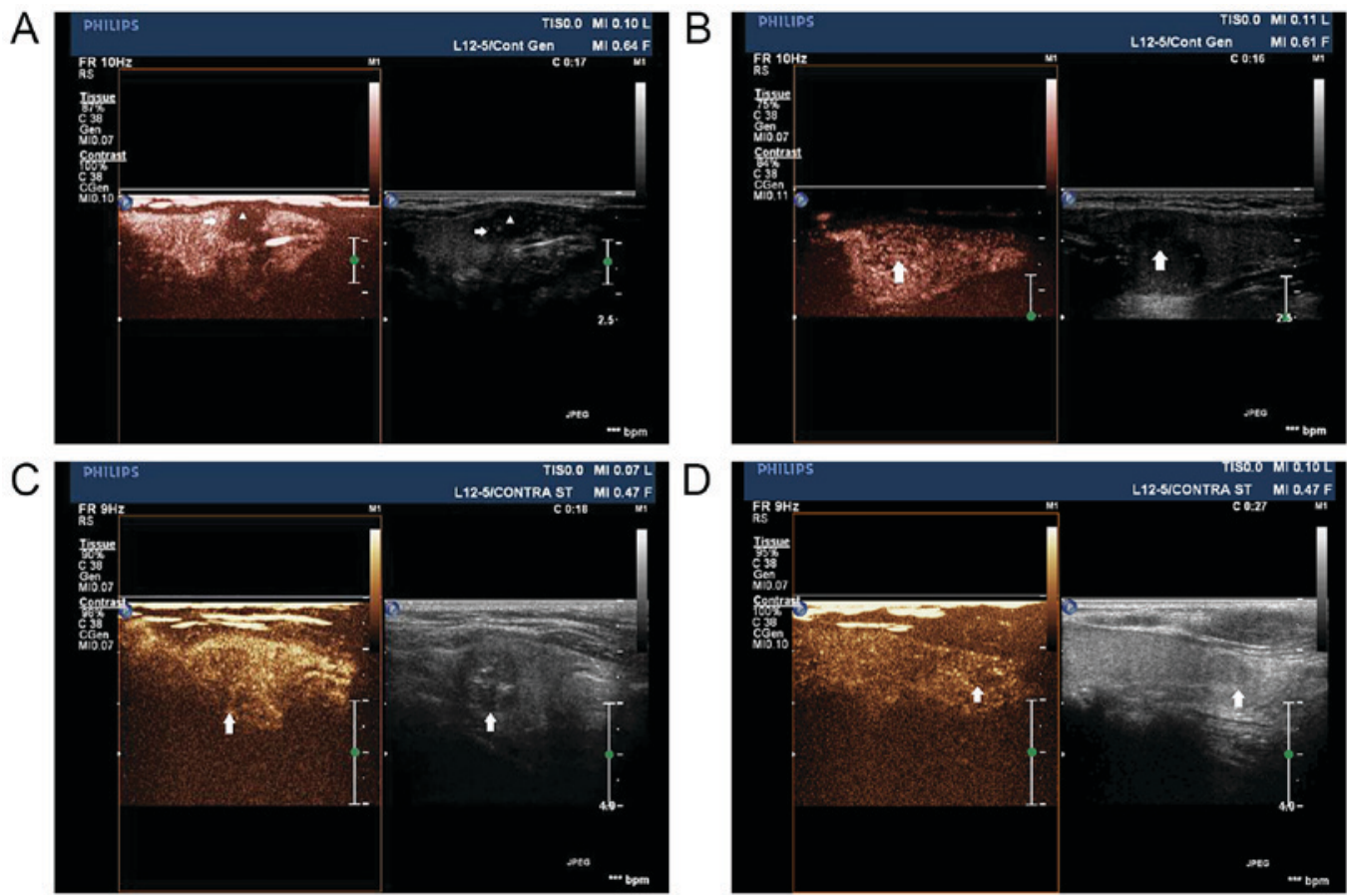

Figure 2. Patterns in early ascending phases of contrast-enhanced ultrasonography. (A) Complex enhancement, manufesting periphery hyperperfused (arrow) with relatively central hypofused (triangle) in a PTC patient with LNM. (B) Pervasive heterogeneous hyper-enhancement (arrow) in a PTC patient with LNM. (C) Centripetal hypo-enhancement (arrow) in a PTC patient without LNM. (D) Iso-enhancement pattern (arrow) in a PTC patient without LNM. PTC, papillary thyroid carcinoma; LNM, lymph node metastasis.

Surgery. The surgical approach at our hospital was as follows: i) Total thyroidectomy (TT) with prophylactic CLND in cases of PTCs with bilateral nodularity and without suspected central neck and/or lateral cervical LNM on US; ii) TT with neck dissection of involved compartments in cases of PTCs with any of following features: suspected central neck and/or lateral cervical LNM on US, cervical LNM confirmed by positive frozen section, extrathyroidal extension, tumor $>4 \mathrm{~cm}$; iii) lobectomy + isthmusectomy (LI) with prophylactic CLND in cases of PTCs without any of following features: contralateral lesions, suspected central neck and/or lateral cervical LNM on US, cervical LNM confirmed by frozen section, extrathyroidal extension.

Demographic data on patient clinical features (sex, age at diagnosis) as well as histological characteristics of tumors and neck lymph samples (size of tumors, lesion locations, extrathyroidal extension) were recorded. All thyroid and lymph node specimens were evaluated by the same pathologist (with 10 years working experience).

MVD measurements. Measurements of MVD were performed independently by the same one pathologist mentioned above, who was blinded to the US findings. Formalin-fixed paraffin-embedded tissues were segmented at 3-4 $\mu \mathrm{m}$ thick sections using a semi-automatic microtome. Consecutive sections were placed on slides pre-coated with egg albumin for routine hematoxylin and eosin staining and on slides coated with poly-L-lysine for immunohistochemical staining for CD34 (a mouse antihuman CD34 monoclonal antibody (W-0117; Dako Corporation, Carpinteria, USA) was used. Completed according to the method by Weidner et al (26), regions with the highest vessel density ('hot spots') were located by scanning the tissue sections under x10-power microscope. Three different fields were randomly chosen within each hot spot, and individual microvessels were manually outlined using freehand draw option in Image Pro Plus software 7.0 (Media Cybernetics, Inc. Singapore, Singapore) and counted under $\mathrm{x} 40$-power power. Images of these selected fields along with the marked microvessels were captured. The mean average scores were tabulated and used for statistical analysis.

Statistical analysis. The SPSS 22.0 software package (IBM, Armonk, NY, USA) was used for statistical analysis. Values are expressed as the mean $\pm \mathrm{SD}$. Categorical variables were analyzed by Fisher's exact test. Continuous variables were compared between groups using the t-test and analysis of variance. Spearman correlation analysis was used to analyze correlation between quantitative variables and LNM. Diagnostic performance was estimated by ROC analysis. A $\mathrm{P}<0.05$ was statistically significant.

\section{Results}

FNA biopsy yielded positive and non-diagnostic results in 39 and 3 patients, respectively, of these 3 patients. Two of the 3 un-diagnostic exhibited extremely weak enhancements on CEUS, while the other a homogeneous hyper-enhancement. Both the enhancement patterns were suggestive of benign, which was ultimately confirmed by surgery. CEUS misdiagnosed 2 PTCs as benign nodules, and 1 subacute thyroiditis as PTC. The sensitivity, specificity and accuracy of CEUS for PTCs suspected by conventional US was 97.4, 75.0, and 95.2\%, respectively. 
Table I. Comparison of clinicopathological characteristics between $\mathrm{LMN}^{+}$group and $\mathrm{LMN}^{-}$group.

\begin{tabular}{|c|c|c|c|}
\hline Variables & $\begin{array}{l}\mathrm{LMN}^{+} \text {group } \\
(\mathrm{n}=27)(\%)\end{array}$ & $\begin{array}{l}\mathrm{LMN}^{-} \text {group } \\
(\mathrm{n}=12)(\%)\end{array}$ & P-value \\
\hline Age (years) & & & $0.012^{\mathrm{a}}$ \\
\hline$\leq 45$ & $21(77.8)$ & $4(33.3)$ & \\
\hline$>45$ & $6(22.2)$ & $8(66.7)$ & \\
\hline Mean age (years) & $41.5 \pm 16.1$ & $49.3 \pm 15.8$ & $0.047^{\mathrm{a}}$ \\
\hline Sex & & & 0.690 \\
\hline Male & 7 (25.9) & $2(16.7)$ & \\
\hline Female & $20(74.1)$ & $10(83.3)$ & \\
\hline Tumor size (mm) & & & $0.041^{\mathrm{a}}$ \\
\hline$\leq 10$ & $8(29.6)$ & $8(66.7)$ & \\
\hline$>10$ & $19(70.4)$ & $4(33.3)$ & \\
\hline Mean size (mm) & $28.1 \pm 10.7$ & $10.5 \pm 3.6$ & $0.039^{\mathrm{a}}$ \\
\hline Right lobe lesion & $13(48.1)$ & $7(58.3)$ & 0.631 \\
\hline Left lobe lesion & $6(22.2)$ & $2(16.7)$ & \\
\hline Multiplicity and/or bilaterality & $8(29.7)$ & $3(25.0)$ & \\
\hline Capsular infiltration (\%) & $17(62.9)$ & $3(25.0)$ & $0.041^{\mathrm{a}}$ \\
\hline Location of the malignancy & & & 0.720 \\
\hline Upper third & $9(33.3)$ & $5(41.6)$ & \\
\hline Mid and lower third & $18(66.7)$ & $7(58.4)$ & \\
\hline
\end{tabular}

${ }^{a}$ P $<0.05$. LMN $^{+}$group, PTC with LNM. LMN` group, PTC without LNM. PTC, papillary thyroid carcinoma; LNM, lymph node metastasis.

A total of 39 cases were confirmed as PTC by surgery. They were divided into two groups: $\mathrm{LNM}^{+}$group (PTCs with LNM, 27 cases) and $\mathrm{LNM}^{-}$group (PTCs without LNM, 12 cases). Table I demonstrates that compared with $\mathrm{LNM}^{-}$ group, patients in $\mathrm{LNM}^{+}$group had a significantly younger age $(\mathrm{P}<0.05)$ and larger mean tumor size $(\mathrm{P}<0.05)$. It was also shown that patients $\leq 45$ years old $(\mathrm{P}<0.05)$, tumors $>10 \mathrm{~mm}$ in size $(\mathrm{P}<0.05)$ and capsular infiltration $(\mathrm{P}<0.05)$ were more commonly seen in $\mathrm{LNM}^{+}$group.

CEUS enhancements in early ascending phases was classified into the following two new patterns for the first time (Fig. 2). The first pattern was early partial hyper-enhancement, presenting either complex enhancement (characterized by periphery hyperperfused with relatively central hypoperfused; Fig. 2A) or pervasive heterogeneous hyper-enhancement (Fig. 2B). The sencond pattern, non hyper-enhancement, including centripetal hypo-enhancement (Fig. 2C) and iso-enhancement (Fig. 2D), both of which were heterogeneous. It was demostrated that, from late ascending to descending phases, PTC lesions mainly presented hypo-enhancement, no stastical differences were found between the two goups. Table II demonstrates that, the ratio of the first enhancement pattern was significantly higher in $\mathrm{LNM}^{+}$group than that in $\mathrm{LNM}^{-}$group $(\mathrm{P}<0.01)$, while the second enhancement pattern was proportionately lower in $\mathrm{LNM}^{-}$group than that in $\mathrm{LNM}^{+}$ group $(\mathrm{P}<0.01)$. Time-intensity curves analysis indicated that MI, IIV and mean intensity ratio of intratumoral/peripheral thyroid parenchyma (MIR) were significantly higher in $\mathrm{LNM}^{+}$ group than that in $\mathrm{LNM}^{-}$group (all $\mathrm{P}<0.05$ ), suggesting higher and faster enhancements were more common during early ascending period in $\mathrm{LNM}^{+}$group. In addition, the measurements of MVD by CD34 expression detection demonstrated that, both intratumoral MVD and MVD ratio of intratumoral/peripheral thyroid parenchyma (MVDR) were higher in $\mathrm{LNM}^{+}$group than that in $\mathrm{LNM}^{-}$group $(\mathrm{P}<0.01)$ (Fig. 3). As regards the Spearman correlation analysis, MI $(r=0.11$, $\mathrm{P}=0.48)$, IIV $(r=0.09, \mathrm{P}=0.35)$, MVDR $(r=0.57, \mathrm{P}<0.05)$ and MIR $(r=0.41, \mathrm{P}<0.05)$ was positively correlated with LNM. The $\mathrm{ROC}$ analysis $(\mathrm{AUC}=0.74, \mathrm{P}<0.05)$ revealed the MIR of 0.86 was the optimal threshold of LNM in PTCs (Fig. 4).

\section{Discussion}

ATA 2015 recommended that, FNA is the accepted standard tool for the evaluation of thyroid nodules, as it is reported to be safe and accurate (9). Definitely, FNA does have limitations, which include a significant rate of non-diagnostic results ranging from 0.6 to $43.1 \%(27,28)$. In total, $7.1 \%$ (3/42) of undiagnosis by FNA were shown in our study. The three cases undiagnosed by FNA alone were diagnosed as benign via combined use of FNA with CEUS, two cases were confirmed for nodular goiter and one for follicular adenoma by surgery ultimately. Nodules of nodular goiter are prone to hemorrhage appearing initially nonechogenic on US. When the blood converts from a liquid state to a solid state, the nonechogenic turns into solid-like hypoechogenic. When US-guided FNA targeted the solid-like hypoechogenic lesions, it's difficult to obtain adequate cellular content. While previous hemorrhage exhibits extremely low enhancement, no enhancement or local non-enhancement, CEUS may prove to be useful in 
Table II. Comparison of CEUS enhancement characteristics and MVD features between between $\mathrm{LMN}^{+}$group and $\mathrm{LMN}^{-}$group.

\begin{tabular}{|c|c|c|c|}
\hline Characteristic & $\begin{array}{l}\mathrm{LMN}^{+} \text {group } \\
(\mathrm{n}=27)(\%)\end{array}$ & $\begin{array}{l}\mathrm{LMN}^{-} \text {group } \\
(\mathrm{n}=12)(\%)\end{array}$ & P-value \\
\hline \multicolumn{4}{|l|}{ Enhancement type } \\
\hline Early partial hyper-enhancement & $22(81.5)$ & $3(25.0)$ & $0.001^{\mathrm{a}}$ \\
\hline $\begin{array}{l}\text { Complex enhancement } \\
\text { Pervasive }\end{array}$ & 17 & 0 & \\
\hline Hyper-enhancement & 5 & 3 & \\
\hline $\begin{array}{l}\text { Non hyper-enhancement } \\
\text { Centripetal }\end{array}$ & $5(18.5)$ & $9(75.0)$ & \\
\hline Hypo-enhancement & 5 & 8 & \\
\hline Iso-enhancement & 0 & 1 & \\
\hline Mean intensity (MI, dB) & $7.7 \pm 4.9$ & $6.4 \pm 3.2$ & $0.033^{\mathrm{b}}$ \\
\hline Intensity increase velocity (IIV, dB/sec) & $1.7 \pm 0.8$ & $1.6 \pm 0.9$ & $0.019^{\mathrm{b}}$ \\
\hline Intensity decrease velocity (IDV, $\mathrm{dB} / \mathrm{sec}$ ) & $1.3 \pm 0.5$ & $1.2 \pm 0.3$ & 0.221 \\
\hline $\begin{array}{l}\text { Mean intensity ratio of Intratumoral/peripheral } \\
\text { thyroid parenchyma (MIR) }\end{array}$ & $0.89 \pm 0.19$ & $0.82 \pm 0.33$ & $0.045^{\mathrm{b}}$ \\
\hline Intratumoral MVD & $5.5 \pm 1.8$ & $5.0 \pm 1.7$ & $0.017^{\mathrm{b}}$ \\
\hline peripheral thyroid parenchyma MVD & $11.2 \pm 1.3$ & $11.6 \pm 1.1$ & 0.095 \\
\hline $\begin{array}{l}\text { MVD ratio of intratumoral/peripheral thyroid } \\
\text { parenchyma (MVDR) }\end{array}$ & $0.42 \pm 0.16$ & $0.48 \pm 0.17$ & $0.021^{\mathrm{b}}$ \\
\hline
\end{tabular}

${ }^{\mathrm{a}} \mathrm{P}<0.01,{ }^{\mathrm{b}} \mathrm{P}<0.05$. LMN+ group, PTC with LNM. LMN- group, PTC without LNM. CEUS, contrast-enhanced ultrasonography; MVD, microvascular density; PTC, papillary thyroid carcinoma; LNM, lymph node metastasis.

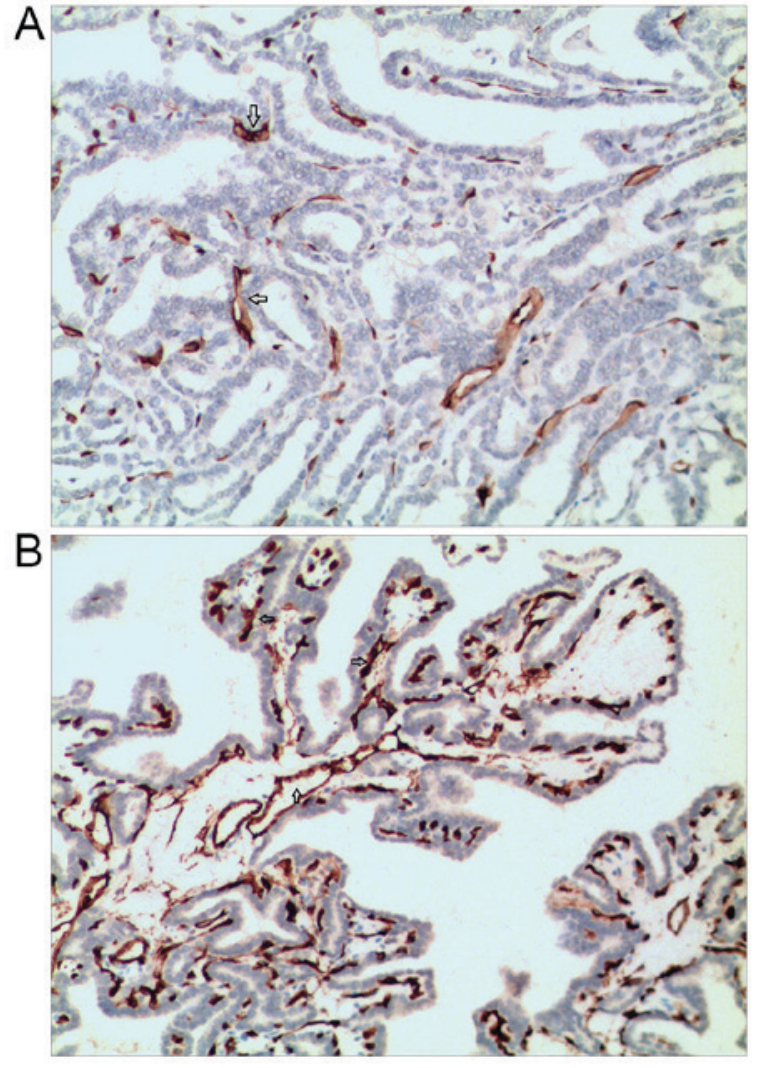

Figure 3. (A and B) CD34 immunohistochemical analysis. Vascular endothelial cells stained brown, $\mathrm{x} 100$-power microscope, showing an increase expression of CD34 in a PTC lesion with LNM (B) than that in a PTC lesion without LNM (A). PTC, papillary thyroid carcinoma. LNM, lymph node metastasis.

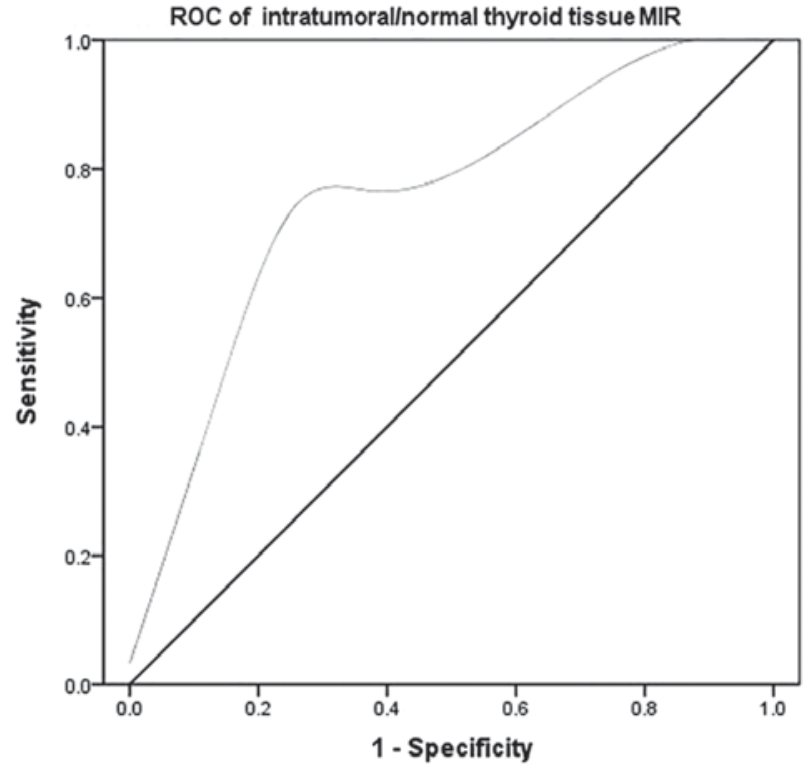

Figure 4. ROC analysis for MIR of intratumoral/peripheral thyroid parenchyma. The area under the ROC curve: $0.74, \mathrm{P}<0.05$. MIR, mean intensity ratio; ROC, receiver operating characteristic.

distinguishing solid-like lesions from true solidary nodules. It is well known that, capsular or vesicular invasion cannot be detected by FNA cytology, so the accuracy of FNA has been largely compromised for follicular lesions, namely follicular thyroid carcinoma (FTC) and follicular adenoma (FA). 
CEUS could provide information of microvascular features in a whole profile for a follicular lesion. It might be one of the possible reasons why the case of follicular adenoma was diagnosed as benign on preoperative CEUS accurately. On the other hand, CEUS misdiagnosed 2 cases of PTC with iso-enhancement as benign nodules, and 1 case of subacute thyroiditis with hypo-enhancement as PTC. CEUS was highly sensitive and accurate in diagnosis of PTC in our study. Its low specificity meant CEUS could not be recommended as a first-line investigation, but a supplement to increase the preoperative diagnostic accuracy of FNA.

LNM is common for PTC patients. Although there is still controversy on the prognosis of LNM, it is universally accepted that cervical LNM should be removed (29,30). Central cervical lymph nodes are easily covered by the thyroid gland, and micro-metastasis of lymph nodes is difficult to detect, so the ability of preoperative US to identify central neck LNM is limited (31). Besides, preoperative US showed a medium sensitivity and a relatively high diagnostic accuracy in detecting lateral neck LNM (32). If PTC patients at high risk of LNM identified preoperatively, they may benifit from extended resection and lymph nodes dissection. For patients with low risk of LNM, conservative treatment with regular monitoring may probably help avoid unnecessary prophylactic CLND and reduce the overall financial costs. Several studies have explored the value of CEUS in the diagnosis of PTC $(23,31)$, however, the application of CEUS of PTC lesions for predicting the risk of LNM has not been reported. The present study compared clinical and pathological characteristics between PTCs with and without LNM, the results showed that $\mathrm{LNM}^{+}$group had a relatively younger mean age and larger mean tumor size compared to $\mathrm{LNM}^{-}$group. Besides, $\mathrm{LNM}^{+}$group had a higher incidence of patients $\leq 45$ years old, tumors $>10 \mathrm{~mm}$ in size and capsular infiltration. These findings which were similar to previous studies $(33,34)$, suggest that age ( $\leq 45$ years old), larger primary tumor size, and the presence of capsular infiltration indicate an increased risk of LNM in PTC.

Studies have shown that, PTC lesions exhibit lower enhancement than benign thyroid nodules on CEUS. As a result of thyroid tumor neovascularization, pathological vessels differ significantly from normal vessels. The lack of muscle and nerve support, vascular dysfunction and tortuosity may lead to low enhancement of the contrast agent $(35,36)$. The present study, for the first time, investigated differences between PTC patients with and without LNM using qualitative and quantitative CEUS. In the present study, PTC lesions mainly presented hypo-enhancement from late ascending to descending phases, no stastical differences were found between PTCs with and without LNM. Yet in early ascending period, enhancements were divided into two patterns (partial hyper-enhancement pattern and non hyper-enhancement pattern) as mentioned in the Results section. By using the newly introduced classification, the ratio of the first pattern in $\mathrm{LNM}^{+}$group was significantly higher than that in $\mathrm{LNM}^{-}$group, supporting the hypothesis that local higher blood supply may be associated with LNM (21). It was revealed that patients in $\mathrm{LNM}^{+}$group had a higher and faster enhancement at early contrast agent ascending period, and had significantly higher MVD than that in $\mathrm{LNM}^{-}$group. PTCs with LNM have been reported to be associated with high expression of vascular proliferative factors, for example vascular growth factor A (VEGF-A) (35). The lymphatic vessel density (LVD), which is directly involved with LNM, is most likely connected to VEGF stimulation $(36,37)$. Besides, VEGF and MVD have significant relationship in assessing microvascular angiogenesis in thyroid carcinomas. Given all that, MVD could be correlated with LNM of PTC (38). Since several studies reported that, quantification of CEUS can be applied to assess MVD in PTC tissues (37,38), CEUS can be recognized as a cost-effective tool for visualizing MVD of PTC. It was investigated that higher local concentrations of microbubbles was observed in PTC lesions with LNM than that without LNM, consistent with the fact that LNM might rely on local rich blood supply. For PTC patients of this study, patial hyper-enhancement on CEUS was speculated to be associated with increased risk of LNM. MIR on quantitative CEUS was positive correlated with LNM in PTC, instead of other quantitative parameters such as MI, IIV, and IDV. The MIR of 0.86 was the optimal threshold of LNM in PTCs. Further researches on a larger population is needed to identify the threshold accurately and help risk-stratify PTC patients with LNM.

\section{Acknowledgements}

We would like to thank Dr Tongtong Zhang and Dr Yu Tang for helpful conversation, Mr Geoff Cunliff and Dr Wenli Hou for helping with language editing.

\section{References}

1. Tian X, Cong M, Zhou W, Zhu J and Liu Q: Relationship between protein expression of VEGF-C, MMP-2 and lymph node metastasis in papillary thyroid cancer. J Int Med Res 36: 699-703, 2008

2. Noguchi S, Murakami N, Yamashita H, Toda M and Kawamoto H: Papillary thyroid carcinoma: Modified radical neck dissection improves prognosis. Arch Surg 133: 276-280, 1998.

3. American Thyroid Association (ATA) Guidelines Taskforce on Thyroid Nodules and Differentiated Thyroid Cancer, Cooper DS, Doherty GM, Haugen BR, Kloos RT, Lee SL, Mandel SJ, Mazzaferri EL, McIver B, Pacini F, et al: Revised American Thyroid Association management guidelines for patients with thyroid nodules and differentiated thyroid cancer. Thyroid 19: 1167-1214, 2009.

4. Gimm O, Rath FW and Dralle H: Pattern of lymph node metastases in papillary thyroid carcinoma. Br J Surg 85: 252-254, 1998.

5. Machens A, Hinze R, Thomusch O and Dralle H: Pattern of nodal metastasis for primary and reoperative thyroid cancer. World J Surg 26: 22-28, 2002.

6. Hundahl SA, Fleming ID, Fremgen AM and Menck HR: A national cancer data base report on 53,856 cases of thyroid carcinoma treated in the U.S., 1985-1995. Cancer 83: 2638-2648, 1998.

7. Mercante G, Frasoldati A, Pedroni C, Formisano D, Renna L, Piana S, Gardini G, Valcavi R and Barbieri V: Prognostic factors affecting neck lymph node recurrence and distant metastasis in papillary microcarcinoma of the thyroid. Results of a study in 445 patients: Thyroid 19: 707-716, 2009.

8. Zhang L, Liu H, Xie Y, Xia Y, Zhang B, Shan G and Li X: Risk factors and indication for dissection of right paraesophageal lymph node metastasis in papillary thyroid carcinoma. Eur J Surg Oncol 42: 81-86, 2016.

9. Haugen BR, Alexander EK, Bible KC, Doherty GM, Mandel SJ, Nikiforov YE, Pacini F, Randolph GW, Sawka AM, Schlumberger M, et al: 2015 American Thyroid Association management guidelines for adult patients with thyroid nodules and differentiated thyroid cancer. Thyroid 26: 1-133, 2016.

10. Wang N, Luo HJ, Yin GB, Dong CR, Xu M, Chen GG and Liu ZM: Overexpression of HIF-2 $\alpha$, TWIST, and CXCR4 is associated with lymph node metastasis in papillary thyroid carcinoma. Clin Dev Immunol 2013: 589423, 2013. 
11. Tang C, Yang L, Wang N, Li L, Xu M, Chen GG and Liu ZM High expression of GPER1, EGFR and CXCR1 is associated with lymph node metastasis in papillary thyroid carcinoma. Int J Clin Exp Pathol 7: 3213-3223, 2014.

12. Machens A, Holzhausen HJ and Dralle H: Skip metastases in thyroid cancer leaping the central lymph node compartment. Arch Surg 139: 43-45, 2004.

13. Roh JL, Kim JM and Park CI: Lateral cervical lymph node metastases from papillary thyroid carcinoma: Pattern of noda metastases and optimal strategy for neck dissection. Ann Surg Oncol 15: 1177-1182, 2008

14. Kouvaraki MA, Shapiro SE, Fornage BD, Edeiken-Monro BS, Sherman SI, Vassilopoulou-Sellin R, Lee JE and Evans DB: Role of preoperative ultrasonography in the surgical management of patients with thyroid cancer. Surgery 134: 946-955, 2003.

15. Caliskan M, Park JH, Jeong JS, Lee CR, Park SK, Kang SW, Jeong JJ, Chung WY and Park CS: Role of prophylactic ipsilateral central compartment lymph node dissection in papillary thyroid microcarcinoma. Endocr J 59: 305-311, 2012.

16. Wada N, Duh QY, Sugino K, Iwasaki H, Kameyama K, Mimura T, Ito $\mathrm{K}$, Takami $\mathrm{H}$ and Takanashi Y: Lymph node metastasis from 259 papillary thyroid microcarcinomas: Frequency, pattern of occurrence and recurrence, and optimal strategy for neck dissection. Ann Surg 237: 399-407, 2003.

17. White ML, Gauger PG and Doherty GM: Central lymph node dissection in differentiated thyroid cancer. World J Surg 31: 895-904, 2007

18. Eloy C, Santos J, Soares P and Sobrinho-Simões M: Intratumoural lymph vessel density is related to presence of lymph node metastases and separates encapsulated from infiltrative papillary thyroid carcinoma. Virchows Arch 59: 595-605, 2011.

19. Lee K, Park DJ, Choe G, Kim HH, Kim WH and Lee HS: Increased intratumoral lymphatic vessel density correlates with lymph node metastasis in early gastric carcinoma. Ann Surg Oncol 17: 73-80, 2010.

20. Folkman J, Merler E, Abernathy C and Williams G: Isolation of a tumor factor responsible for angiogenesis. J Exp Med 133: 275-288, 1971 .

21. Zhan WW, Zhou P, Zhou JQ, Xu SY and Chen KM: Differences in sonographic features of papillary thyroid carcinoma between neck lymph node metastatic and nonmetastatic groups. J Ultrasound Med 31: 915-920, 2012.

22. Hakala T, Sand J, Kellokumpu-Lehtinen PL, Huhtala H, Leinonen R and Kholová I: Recurrent thyroid cancers have more peritumoural lymphatic vasculature than nonrecurrent thyroid cancers. Eur J Clin Invest 44: 825-832, 2014.

23. Chen M, Zhang KQ, Xu YF, Zhang SM, Cao Y and Sun WQ: Shear wave elastography and contrast-enhanced ultrasonography in the diagnosis of thyroid malignant nodules. Mol Clin Oncol 5: 724-730, 2016

24. Piscaglia F, Nolsøe C, Dietrich CF, Cosgrove DO, Gilja $\mathrm{OH}$, Bachmann Nielsen M, Albrecht T, Barozzi L, Bertolotto M, Catalano $\mathrm{O}$, et al: The EFSUMB guidelines and recommendations on the clinical practice of contrast enhanced ultrasound (CEUS): Update 2011 on non-hepatic applications. Ultraschall med 33: 33-59, 2012.

25. Rosário PW, de Faria S, Bicalho L, Alves MF, Borges MA, Purisch S, Padrão EL, Rezende LL and Barroso AL: Ultrasonographic differentiation between metastatic and benign lymph nodes in patients with papillary thyroid carcinoma. J Ultrasound Med 24: 1385-1389, 2005.
26. Weidner N, Semple JP, Welch WR and Folkman J: Tumor angiogenesis and metastasis-correlation in invasive breast carcinoma. N Engl J Med 324: 1-8, 1991.

27. Ceresini G, Corcione L, Morganti S, Milli B, Bertone L, Prampolini R, Petrazzoli S, Saccani M, Ceda GP and Valenti G: Ultrasound-guided fine-needle capillary biopsy of thyroid nodules, coupled with on-site cytologic review, improves results. Thyroid 14: 385-389, 2004

28. Tabaqchali MA, Hanson JM, Johnson SJ, Wadehra V, Lennard TW and Proud G: Thyroid aspiration cytology in Newcastle: A six year cytology/histology correlation study. Ann R Coll Surg Engl 82: 149-155, 2000.

29. Hay ID, Thompson GB, Grant CS, Bergstralh EJ, Dvorak CE, Gorman CA, Maurer MS, McIver B, Mullan BP, Oberg AL, et al: Papillary thyroid carcinoma managed at the Mayo Clinic during six decades (1940-1999): Temporal trends in initial therapy and long-term outcome in 2444 consecutively treated patients. World J Surg 26: 879-885, 2002

30. Yamashita H, Noguchi S, Murakami N, Kawamoto $H$ and Watanabe S: Extracapsular invasion of lymph node metastasis is an indicator of distant metastasis and poor prognosis in patients with thyroid papillary carcinoma. Cancer 80: 2268-2272, 1997.

31. Xiang D, Hong Y, Zhang B, Huang P, Li G, Wang P and Li Z: Contrast-enhanced ultrasound (CEUS) facilitated US in detecting lateral neck lymph node metastasis of thyroid cancer patients: Diagnosis value and enhancement patterns of malignant lymph nodes. Eur Radiol 24: 2513-2519, 2014.

32. Na DK, Choi YJ, Choi SH, Kook SH and Park HJ: Evaluation of cervical lymph node metastasis in thyroid cancer patients using real-time CT-navigated ultrasonography: Preliminary study. Ultrasonography 34: 39-44, 2015.

33. Lang BH, Lo CY, Chan WF, Lam KY and Wan KY: Staging systems for papillary thyroid carcinoma: A review and comparison. Ann Surg 245: 366-378, 2007.

34. Qu N, Zhang L, Ji QH, Chen JY, Zhu YX, Cao YM and Shen Q: Risk factors for central compartment lymph node metastasis in papillary thyroid microcarcinoma: A meta-analysis. World J Surg 39: 2459-2470, 2015.

35. Meng XY, Zhang Q, Li Q, Lin S and Li J: Immunohistochemical levels of cyclo-oxygenase-2, matrix metalloproteinase-9 and vascular endothelial growth factor in papillary thyroid carcinoma and their clinicopathological correlations. J Int Med Res 42: 619-627, 2014

36. Sun XF and Zhang H: Clinicopathological significance of stromal variables: Angiogenesis, lymphangiogenesis, inflammatory infiltration, MMP and PINCH in colorectal carcinomas. Mol Cancer 5: 43, 2006.

37. Dufort S, Sancey L, Hurbin A, Foillard S, Boturyn D, Dumy P and Coll JL: Targeted delivery of a proapoptotic peptide to tumors in vivo. J Drug Target 19: 582-588, 2011.

38. Yu XM, Lo CY, Lam AK, Leung P and Luk JM: Serum vascular endothelial growth factor $\mathrm{C}$ correlates with lymph node metastases and high-risk tumor profiles in papillary thyroid carcinoma. Ann Surg 247: 483-489, 2008. 